\title{
Lifetime Exposure to Adverse Events and Reinforcement Sensitivity in Obsessive-Compulsive Prone Individuals
}

\author{
Grazia Ceschi,' Melissa Hearn,' Joël Billieux,' and Martial Van der Linden',2 \\ I Department of Psychology, University of Geneva, Switzerland \\ 2 Cognitive Psychopathology Unit, University of Liège, Belgium
}

\begin{abstract}
A diathesis-stress perspective of obsessive-compulsive symptoms (OCS) predicts that exposure to adverse events and personality dispositions jointly influence OCS. Gray and McNaughton's (2000) model of personality posits that, faced with challenging circumstances, individuals with a high sensitivity to punishment (SP) will be more prone to OCS because they cannot avoid the downward spiral into anxiety. The current study investigates OCS severity in relation to lifetime exposure to adverse events (AE), SP, and sensitivity to reward (SR) in 122 nonclinical adults. The results indicate that OCS severity is predicted by AE, SP and SR. Interestingly, the impact of adverse experiences is moderated by SR and not SP. These findings suggest that: (1) exposure to adverse events and SP are independent OCS risk factors, and (2) exposure to adverse events is more critical for reward dependent people. This is discussed in light of responsibility and 'not just right experiences' in OCS, along with the role of impulsivity in the obsessivecompulsive disorder spectrum.
\end{abstract}

Keywords: negative life events, obsessive-compulsive disorder, anxiety, impulsivity, reward drive, diathesis-stress model

A diathesis-stress model of obsessive-compulsive symptoms (OCS) would posit that maladaptive beliefs (e.g., heightened sense of responsibility) interact with negative life events in the onset and development of obsessive-compulsive reactions (Dohrenwend \& Dohrenwend, 1981). This theoretical perspective dates back to Bleuler and Freud (1893-1895), who described the emergence of OCS in a person suffering from 'post-traumatic hysteria'. Yet, in more recent studies on OCS, situational factors have often been neglected in favor of cognitive appraisal dysfunctions (e.g., Myers, Fisher, \& Wells, 2009).

A small number of recent publications have suggested a positive relationship between traumatic exposure and OCS severity. For instance, Cromer, Schmidt, and Murphy (2007b) examined the contribution of challenging life circumstances in the expression of OCS in 265 obsessive-compulsive disorder (OCD) patients, 54\% of whom endorsed at least one traumatic life experience. The association between trauma and OCS severity remained significant even after age, OCD age-of-onset,

Address for correspondence: Grazia Ceschi, Department of Psychology, FPSE, University of Geneva, $40 \mathrm{Bd} d u$ Pont d'Arve, CH-1205 Geneva, Switzerland. E-mail: grazia.ceschi@unige.ch 
comorbidity, and depression were accounted for. These findings were replicated in other OCD samples (Cromer, Schmidt, \& Murphy, 2007a), in OCS prone nonclinical individuals (Coles \& Horng, 2006; Coles, Pietrefesa, Schofield, \& Cook, 2008), as well as in patients suffering from post-traumatic stress disorder (PTSD; Sasson et al., 2005; Tuerk, Grubaugh, Hamner, \& Foa, 2009). The same conclusions were drawn for different types of negative life incidents, from extremely upsetting events in adulthood (e.g., high-combat experiences; De Silva \& Marks, 2001; Tuerk et al., 2009) to childhood abuse and neglect (Lochner et al., 2002; Mathews, Kaur, \& Stein, 2008). Even if more equivocal results have also been published (Grabe et al., 2008; Huppert et al., 2005), the data are generally supportive of the assumption that adverse life events tend to be associated with an increase in OCS severity. While this association has been found to be moderate in size (correlations ranging from .14 to .23; Coles \& Horng, 2006; Coles et al., 2008; Cromer et al., 2007a, 2007b), its crucial role has been incorporated in the most influential cognitive models of OCD (Rachman, 1997; Salkovskis, 1999). In accordance with the diathesis-stress perspective, these models postulate that adverse events do not directly lead to OCS, but rather indirectly through cognitive appraisal.

In the last 20 years, a diversity of OCD-specific maladaptive appraisals has been identified. These include a heightened sense of responsibility (Salkovskis, Shafran, Rachman, \& Freeston, 1999) or a tendency toward perfectionism (Frost \& Steketee, 1997). OCD patients have been shown to score high not only on OCDspecific maladaptive appraisals, but also on more general cognitive-evaluation biases related to, for instance, neuroticism (Samuel et al., 2000), or trait anxiety (HoehnSaric, McLeod, \& Hipsley, 1995). This is in accordance with the presupposition that people suffering from OCS show an anxiety bias in information processing in which events are appraised as overly threatening (Nestadt et al., 2001).

This well-established conceptualisation of OCD as an anxiety disorder has recently been challenged (Storch, Abramowitz, \& Goodman, 2008). It has been suggested that OCS may be associated with an inability to inhibit a repetitive dominant response (e.g., checking, washing) that is better captured by impulsivity (Bartz \& Hollander, 2006). In agreement with this position, OCD patients compared with individuals suffering from other forms of anxiety presented a specific deficit in the capacity to inhibit a prepotent response (Van der Linden, Ceschi, Zermatten, Dunker, \& Perroud, 2005). Moreover, OCD patients (especially tic-prone patients) reported a higher score of impulsivity compared to patients presenting other anxiety disorders (Summerfeldt, Hood, Antony, Richter, \& Swinson, 2004). However, a recent study of 330 undergraduate students provided only partial support to the association between OCS and impulsivity (Sulkowski et al., 2009). Analogous mixed findings were also published by Ray Li and Chen (2007). Given the difficulty defining the construct of impulsivity, these inconsistencies might be accounted for by the heterogeneity of impulsivity definitions and measures used in studies. In sum, the literature supports a diathesis-stress model of OCS whereby the role of adverse events may be modulated by anxious and, possibly, impulsive dispositions.

Impulsivity is a multidimensional construct composed of several dimensions (e.g., Enticott \& Ogloff, 2006) supported by specific executive and motivational mechanisms (e.g., Gay, Rochat, Billieux, d'Acremont, \& Van der Linden, 2008). In the context of OCS, at least two levels of impulsivity deserve attention. The first level is motivational (e.g., Elliot $\&$ Thrash, 2002) and refers to constructs such as approach behaviours (e.g., sensitivity to reward, behaviour activation system, posi- 
tive affects, weak fear system, extraversion) and withdrawal behaviours (e.g., sensitivity to punishment, behaviour inhibition system, negative affects, activated fear system, neuroticism). The second level is executive and concerns self-control capacities based on executive processes (e.g., prepotent response inhibition, cognitive switching ability). In this perspective, Dawe, Gullo, and Loxton (2004) indicate that impulsivity-related behaviours often rely upon either a reward drive (i.e., the sensitivity to unconditioned and conditioned reward stimuli, which mainly relate to automatic-motivational processes), or a rash impulsivity (i.e., the tendency to engage in rash and spontaneous behaviours associated with a lack of consideration of future consequences, which mainly depends on controlled-executive processes). Taking into account this distinction, it appears that the majority of studies that found a relationship between impulsivity and OCS have used measures that better capture the rash impulsiveness dimension of impulsivity rather than its reward drive dimension (e.g., Summerfeldt et al., 2004; Van der Linden et al., 2005; Zermatten \& Van der Linden, 2008). In fact, the relationship between OCS and reward drive remains poorly investigated.

In this context, Gray's personality model, which distinguishes between anxiety driven and impulsivity driven attitudes is of interest for further exploring the relationships between OCS and reward drive-related (or motivational) aspects of impulsivity (Gray \& McNaughton, 2000). The two main dimensions of Gray's model reflect individual differences in sensitivity to punishment (SP) and sensitivity to reward (SR). The SP, which taps into the construct of the 'Behavioural Inhibition System' (BIS), is associated with anxiety and acquires behavioural control in response to danger, frustration, and/or novelty. The SR, which taps into the construct of the 'Behavioural Activation System' (BAS), is associated with the control of approach behaviours induced by events evaluated as rewarding. The SR can therefore be related to motivational aspects of impulsivity. The studies of interindividual differences in sensitivity to reinforcement benefitted from the recent development of self-report measures of Gray's constructs, such as the Sensitivity to Punishment and Sensitivity to Reward Questionnaire (SPSRQ; Torrubia, Avila, Molto, \& Caseras, 2001).

Two recent studies have used the SPSRQ in OCD patients and OCS-prone individuals (Fullana, Mataix-Cols, Caseras et al., 2004; Fullana, Mataix-Cols, Trujillo et al., 2004). In accordance with Gray's predictions, Fullana and colleagues showed that individuals with subclinical OCS $(N=25)$ and OCD patients $(N=56)$ reported a significantly higher SP level than controls. These results remain unchanged even after controlling for depression and anxiety. A closer look at these findings (Fullana, Mataix-Cols, Trujillo et al., 2004) reveals a small increase in SR in both OCS samples compared to their respective controls. However, this difference did not reach an acceptable level of significance, possibly because of a lack of statistical power. This same conclusion also applied to a SPSRQ dataset of 56 OCD-hoarding patients (Fullana, Mataix-Cols, Caseras et al., 2004). In summary, only a few studies evaluate Gray's personality dimensions in OCS-prone individuals. These studies corroborate the assumption that OCS is associated with a marked SP, suggesting an anxiety disposition. However, they remain inconclusive concerning the impulsivity disposition accounted for by SR. Yet, it cannot be excluded that this latter questionable finding is due to methodological limits, for instance, the sample size or the binomial, instead of the dimensional assessment of SR that could have underestimated the importance of the phenomenon. Moreover, SP and SR have not yet been studied in the framework

\section{Behaviour Change}


of a diathesis-stress model of OCS, leaving unexplored the role of sensitivity to reinforcement on emotional outcome after traumatic experiences.

In accordance with a diathesis-stress model of OCD, the current study examined the relationship between trauma and OCS in a community sample by assuming that this relationship is moderated by SP. A nonclinical sample of undergraduate students was selected. Studies show that obsessions and compulsions are normal phenomena occurring in nonclinical populations (Muris, Merckelbach, \& Clavan, 1997). Thus, community samples are commonly considered valid analogues of OCD patients (Mataix-Cols, Vallejo, \& Sanchez-Turet, 2000).

Our research extends Fullana and colleague's studies (2004) in several ways. First, a new version of the SPSRQ scale developed by Lardi, Billieux, d'Acremont, and Van der Linden (2008) was used. The new version removed 13 problematic items from the original version by Torrubia et al. (2001) and asked participants to report their agreement to items on 4-point scales instead of replying with a Yes-No answer. Second, the whole population variance was considered by computing intraindividual instead of intergroup effects. Finally, a severity score for lifetime adverse event exposure was calculated.

By addressing these methodological issues, the current study considered the following predictions. First, in accordance with previous studies we expected a correlation between trauma and OCS severity (i.e., Coles \& Horng, 2006; Coles et al., 2008; Cromer et al., 2007a, 2007b), and between SP and OCS severity (i.e., Fullana et al., 2004). Second, in line with previous data on impulsivity in OCS (i.e., Summerfeldt et al., 2004; Van der Linden et al., 2005; Zermatten et al., 2008 ), we also expected a correlation between SR and OCS severity. Finally, in accordance with a diathesis-stress model of OCS (i.e., Rachman, 1997; Salkovskis, 1999), we anticipated that SP and/or SR would moderate the relation between trauma and OCS severity above and beyond the variance explained by depression.

\section{Method}

\section{Population}

The current sample included 122 undergraduate students (107 females) from the University of Geneva. Participants ranged from 18 to 45 years of age, with a mean age of 22.93 years $(S D=4.76)$. All were fluent French speakers and volunteered to participate in the study for course credit.

\section{Measures}

Lifetime exposure to adverse events (Posttraumatic Diagnostic Scale - French adaptation; PDS-F; Hearn, Ceschi, Brillon, $\mathcal{E}$ Van der Linden, 2010). The PDS-F is an extension of the trauma checklist of the Posttraumatic Diagnostic Scale (PDS; Foa, Cashman, Jaycox, \& Perry, 1997). The PDS presents a trauma checklist of 11 very distressing incidents (i.e., serious accident, sexual assault, life-threatening illness) and 1 open item. The PDS-F checklist includes 25 stressful events extending the original PDS checklist with 14 supplementary adverse events (i.e., difficult childbirth, abortion or miscarriage, divorce, separation of parents). These supplementary events were incorporated in the PDS-F from a catalog of residual traumatic events reported in previous studies by a sample of nonclinical participants (David, Ceschi, Billieux, \& Van der Linden, 2008). In response to each adverse event, participants were asked to rate its lifetime frequency on a 4-point scale ranging from 0 
(Not experienced) to 3 (Experienced 3 times or more), and its subjective degree of stress on an 8 point-scale ranging from 0 (Not stressful at all) to 7 (Extremely stressful). For each event, a severity score was calculated by multiplying event frequency by stress intensity. A mean value per participant was computed by averaging severity scores across endorsed events. In our sample, the PDS-F presented good internal consistency $(\alpha=.71)$.

Obsessive-compulsive severity: Obsessive-Compulsive Inventory - Revised (OCIR; Foa et al., 2002); French version by Zermatten, Van der Linden, Jermann, and Ceschi (2006). The OCI-R includes 18 items describing a variety of OCS. By reference to each item, participants are asked to report their degree of distress on a 5 point scale ranging from 0 (Not at all) to 4 (Extremely). The psychometric properties of the original English (Foa et al., 2002) and French (Zermatten et al., 2006) versions of the OCI-R are excellent. In our study, the OCI-R presented good internal consistency $(\alpha=.86)$.

Depression: Beck Depression Inventory, version 2 (BDI-II; Beck, Steer, Ë Brown, 1998). The 21-item BDI-II inventory provides a self-report measure of depressed mood. The psychometric qualities of BDI-II have been widely demonstrated (Beck et al., 1998). BDI-II presented an excellent internal consistency $(\alpha=.91)$ in our sample.

Sensitivity to Punishment and Sensitivity to Reward. (SPSRQ; Torrubia et al., 2001; short French version by Lardi et al., 2008). The short SPSRQ version is a 35-item questionnaire elaborated on Gray's personality model (Gray \& McNaughton, 2000). It consists of two subscales, the Sensitivity to Punishment scale (SP), and the Sensitivity to Reward scale (SR). The 18 items of the SP subscale were developed to assess behavioural inhibition in situations involving the possibility of aversive consequences and threats (i.e., 'Do you prefer not to ask for something when you are not sure you will obtain it?'). The 17 items of the SR subscale capture sensitivity to signals of reward in relation to approach behaviours and hedonism (i.e., 'Does the good prospect of obtaining money motivate you strongly to do some things?'). Each item was rated on a 4-point scale, ranging from 1 (No) to 4 (Yes). SP and SR scores were obtained by summing corresponding item values. The English and French versions of the SPSRQ showed good reliability and validity (Lardi et al., 2008). Good internal consistency was established for both subscales in our sample (SP: $\alpha=.87$; SR: $\alpha=.80$ ).

\section{TABLE 1}

Cronbach's $\alpha$, Means and Standard Deviations for Study Variables, and Zero-Order Pearson's Correlations Among Them

\begin{tabular}{lccccccc} 
& $\alpha$ & $N$ valid & $M(S D)$ & $(1)$ & $(2)$ & (3) & (4) \\
\hline 1. Adverse events & .71 & 122 & $1.905(1.284)$ & - & & & \\
2. Depression & .91 & 114 & $8.430(7.862)$ & $.197^{*}$ & - & & \\
3. Sen. Reward & .80 & 116 & $37.108(7.106)$ & .065 & .144 & - & \\
4. Sen. Punishment & .87 & 116 & $43.457(9.032)$ & .108 & $.444^{* *}$ & .144 & - \\
5. OCl-R & .86 & 116 & $15.310(9.412)$ & $.240^{* *}$ & $.286^{* *}$ & $.360^{* *}$ & $.346^{* *}$ \\
\hline Note: $N=122$ (pairwise valid $108<n<116) ;{ }^{*} p<.05 ;{ }^{* *} p<.01$ & & & &
\end{tabular}




\section{Results}

As indicated in Table 1 , the number of valid cases differed between variables $(n=$ 114-122). Thus, missing data were excluded pairwise. Preliminary statistical analyses showed that basic assumptions were well-respected without further need of data transformation, except for the $z$ score conversion recommended for the calculation of interaction terms.

Table 1 displays zero-order Pearson's correlations between adverse events, depression, SR, SP and OCS. As expected, individuals reporting a greater number of adverse events also tend to endorse more severe OCS, $r(116)=.240, p=.005$.

A hierarchical regression analysis was used to predict OCI-R as a function of dispositional (SP, SR) and situational factors (PDS-F) as well as their interactions. The variables were forced into the regression in 4 steps. Given the strong association of depression with SP and SR, depression was controlled by entering the BDI-II score in step 1. The two dispositional factors were forced into the regression in step 2, and the lifetime exposure to adverse events in step 3. In the final step, the two interaction terms between exposure to adverse events and each dispositional score (SP, SR) were added to assess moderation effects.

As illustrated in Table 2, the regression showed that OCS severity is significantly predicted by SP and SR, even after having partialised the variance accounted for by depression. More precisely, results from step 2 reveal that SP and SR jointly predicted a significant amount of variance in the OCI-R, $\left(\Delta R^{2}=.151 ; F(2,104)=\right.$ $10.215 ; p=.000)$. Individuals who reported a higher SP level $(\beta=.243 ; t=2.519$; $p=.013)$ as well as a higher SR level $(\beta=.306 ; t=3.511 ; p=.001)$ were also those who suffered from more severe OCS. The third regression step proved that exposure to adverse events makes an additional contribution in the prediction of OCS

\section{TABLE 2}

Hierarchical Regression Analyses Predicting OCS Severity by Control Variable (Depression) and Predictors (Sensitivity to Punishment, Sensitivity to Reward, Exposure to Adverse Events, and Their Interactions)

\begin{tabular}{llccccc}
\hline $\begin{array}{l}\text { Dependent } \\
\text { Variable OCI-R }\end{array}$ & Predictors & Beta & $t$ & Sig. & $R^{2}$ & $R^{2}$ Change \\
\hline Step 1 & Depression & .286 & 3.068 & .003 & $.082^{* *}$ & \\
Step 2 & Depression & .134 & 1.391 & .167 & $.232^{* *}$ & $.151^{* *}$ \\
& Sen. reward & .306 & 3.511 & .001 & & \\
Step 3 & Sen. punishment & .243 & 2.519 & .013 & & \\
& Depression & .102 & 1.061 & .291 & $.262^{* *}$ & $.029^{*}$ \\
& Sen. reward & .300 & 3.488 & .001 & & \\
Step 4 & Sen. punishment & .266 & 2.756 & .007 & & \\
& Adverse events & .239 & 2.516 & .013 & & \\
& Depression & .063 & 0.666 & .507 & $.311^{* *}$ & $.050^{*}$ \\
& Sen. reward & .276 & 3.268 & .001 & & \\
& Sen. punishment & .268 & 2.880 & .005 & & \\
& Adverse events & .167 & 1.975 & .051 & & \\
& AE ${ }^{*}$ SP & .030 & 0.359 & .720 & & \\
& AE ${ }^{*}$ SR & .222 & 2.607 & .011 & & \\
\hline
\end{tabular}

Note: ${ }^{*} p<.01 ; * p<.05 ; \mathrm{AE}=$ adverse events; $\mathrm{SP}=$ sensitivity to punishment; $\mathrm{SR}=$ sensitivity to reward. $N=122$, pairwise case deletion. 


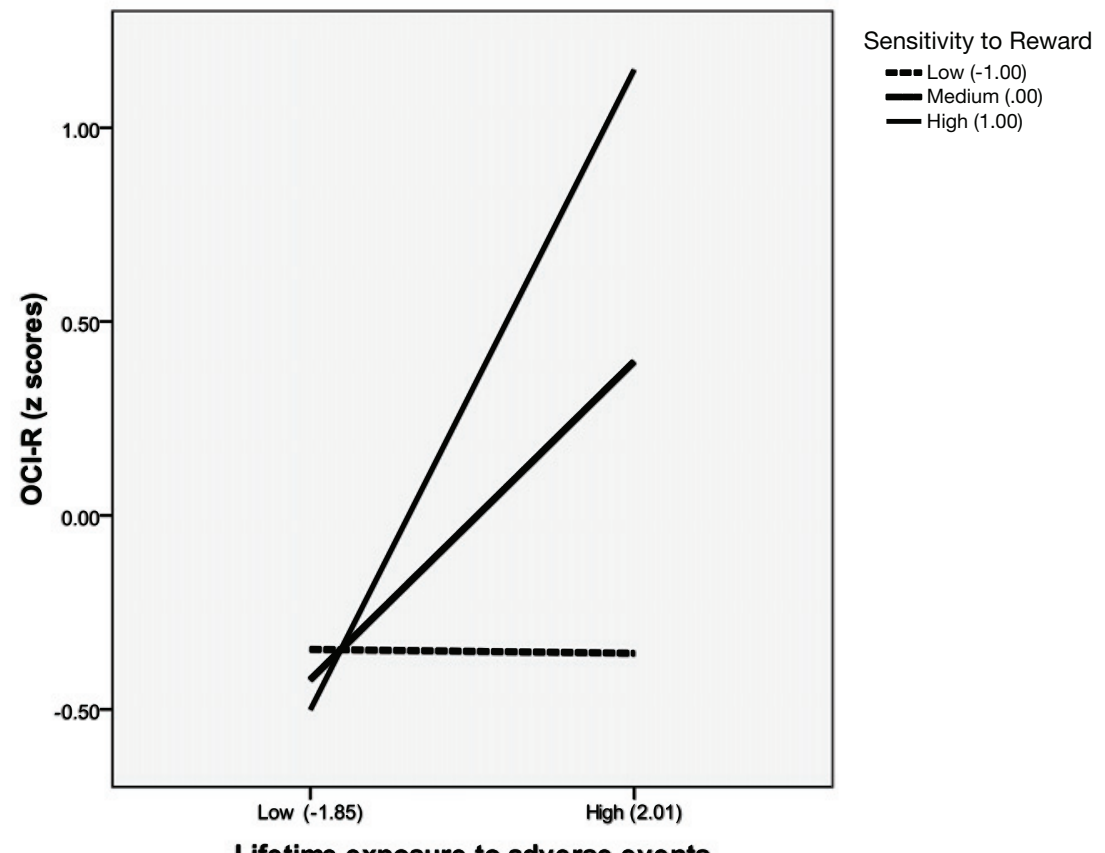

Lifetime exposure to adverse events

\section{FIGURE 1}

Effect between sensitivity to reward and lifetime exposure to adverse life events on OCS severity.

severity $\left(\Delta R^{2}=.029 ; F(1,103)=4.087 ; p=.046\right)$ and suggests that individuals encountering more adverse events tend to suffer from more severe OCS $(\beta=.175$; $t=2.022 ; p=.046)$ independently of their reinforcement sensitivity and level of depression.

The interaction terms entered in the final equation made a supplementary contribution in predicting OCS severity $\left(\Delta R^{2}=.050 ; F(2,101)=3.652 ; p=.029\right)$. In this model, OCS severity was predicted by lifetime exposure to adverse events $(\beta=.167$; $t=1.975 ; p=.051), \operatorname{SP}(\beta=.268 ; t=2.880 ; p=.005)$, and SR $(\beta=.276 ; t=3.268$; $p=.001)$. Furthermore, the interaction effect between SR and adverse events contributed significantly to explain variance in OCS severity $(\beta=.222 ; t=2.607$; $p=.011)$.

Figure 1 shows that the exposure to adverse events did not result in any residual change in OCS severity for those reporting a low SR $(\beta=-.003 ; t=-.021$; $p=.983)$. On the contrary, for individuals who reported a marked SR, exposure to adverse events was correlated with OCS severity with an increasing standardized simple slope of $\beta=.205(t=2.453 ; p=.016)$ and $\beta=.413(t=3.661 ; p=.000)$ for participants reporting a medium and high SR level, respectively.

\section{Discussion}

The current study investigated the role of adverse life events on OCS expression within a diathesis-stress model that considers the moderation effects of two core

\section{Behaviour Change}

Downloaded from https:/www.cambridge.org/core. University of Basel Library, on 30 May 2017 at 16:29:06, subject to the Cambridge Core terms of use, 
personality dispositions formulated by Gray et al. (2000): SP and SR. In accordance with previous studies, OCS severity was predicted by the lifetime exposure to adverse events (Coles \& Horng, 2006; Coles et al., 2008; Cromer et al., 2007a, 2007b), and sensitivity to punishment (Fullana et al., 2004). Thus, individuals who encounter frequent and distressing experiences, like those reporting a pronounced sensitivity to punishment, are at risk to develop OCS. Contrary to a diathesis-stress model of OCS, the present study shows, however, that SP does not enhance the impact of adverse life events. Accordingly, an additive rather than a multiplicative model seems to be a more accurate description of OCS variance as a function of adverse events exposure and SP. Despite this observation, a diathesis-stress model of OCS was found to be an acceptable approximation of OCS variance as a function of SR disposition. It is worth nothing that exposure to adverse life events predicts OCS severity particularly for individuals reporting an elevated SR, namely those motivated by incentive and reward. These findings are discussed in four points.

First, the current data indicate that the lifetime exposure to adverse events predicts OCS severity. This relation is moderate in size, but compatible with previous evidence (Coles \& Horng, 2006; Coles et al., 2008; Cromer et al., 2007a, 2007b). We extended previous findings by showing that exposure to adverse events predicts residual change in OCS severity above and beyond the variance accounted for by depression and reinforcement sensitivity. Given OCS heterogeneity (Mataix-Cols, Rosario-Campos, \& Leckman, 2005), a more rigorous distinction of OCS profiles in further studies may be useful for identifying more robust OCS-trauma associations. For instance, Cromer and colleagues (2007b) found that the occurrence of traumatic experiences was linked to checking and ordering but not to other OCS subtypes.

Second, the current data on SP fit an additive model of OCS whereby the anxiety disposition (captured by a high SP score) predicts OCS regardless of event exposure. Likewise, a heightened exposure to adverse life events may constitute a vulnerability factor of OCS independently of individual anxiety dispositions. In other words, OCS prevails in individuals with a high SP whether or not they have been exposed to adverse events. Thus, the current study suggests that individuals reporting a high SP score, even if motivated to avoid threatening situations, tend to focus their attention on future events that could be threatening regardless of the likelihood of their occurrence. Thus, SP does not seem to be instrumental in controlling the uncertainty of possible adverse consequences. Similar unpredicted conclusions have been described for other anxious cognitions such as 'worries' (Freeston, Rhéaume, Letarte, Dugas, \& Ladouceur, 1994) or 'intolerance to uncertainty' (Dugas, Buhr, \& Ladouceur, 2004). These anxious cognitions have been found to be inefficient in moderating the impact of stressful events on emotional adjustment (Dugas \& Robichaud, 2007). This result is consistent with Fullana et al.'s (2004) findings and indicates that a high SP should be considered a vulnerability factor for OCS, independently of life experiences.

Third, the current study indicates that among individuals who reported moderateto-high SR scores, exposure to adverse events is associated with more severe OCS. Conversely, adverse events do not result in any residual change in OCS severity for those with low SR scores. These data conflict with results published by Fullana's group, based on evaluations of between-subgroup differences. These studies suggested that OCD subgroups did not endorse more SR than their matched controls (Fullana, Mataix-Cols, Caseras et al., 2004; Fullana, Mataix-Cols, Trujillo et al., 2004). A closer look to Fullana's findings indicates, however, that SR scoring tended to differentiate 
the subgroups. Congruent to the current results, Fullana et al.'s OCD-prone subgroups have a propensity to report more pronounced sensitivity to reward than other persons. Some methodological limitations (i.e., sample size, suboptimal SR scale, unidentified psychometric properties of SR) could have deflated the estimation of SR in Fullana et al.'s studies. Although additional replication is needed to establish robust conclusions, our finding already reveals the importance of impulsivity, and more specifically its reward-driven component, in the understanding of OCS.

Previous studies on impulsivity in OCS have mainly focused on rash impulsiveness, namely the inability to inhibit prepotent responses promoting compulsive behaviours (e.g., Van der Linden et al., 2005). Few studies have assessed a reward drive attitude compatible with Gray and McNaughton' (2000) 'Behaviour Activation System' or Torrubia et al.'s (2001) SR scale. Our findings argue that OCS can be related to the reward drive level of impulsivity activated by a context of adverse experiences. In accordance with this suggestion, scholars have recently begun to recognise that aspects of motivation, such as a sense of 'incompleteness', accompanied the 'not just right experience', may be important in explaining different aspects of the compulsive behaviours. 'Incompleteness' is defined as 'an inner sense of imperfection, connected with the perception that actions or intentions have been incompletely achieved' (Pietrefesa \& Coles, 2008; p. 224). Recently, Pietrefesa and Coles (2008) provided strong support to the assumption that, in addition to harm avoidance, incompleteness has to be considered a key motivation underlying compulsive behaviour in OCD, especially in association with the selfprescribed aspects of perfectionism. This is in line with the long-established clinical observation that OCD patients present an inflated sense of responsibility and a desire for perfectionism (Rachman, 1993; Salkovskis et al., 1999). For Salkovskis (1999), beliefs of responsibility involve socialisation processes that favour the development of an ideal, perfect self, and that trauma undermines confidence and increases feelings of guilt. The current study suggests that the heightened sense of responsibility and the 'not just right experience' commonly described in OCD can be explained as a motivation to compensate a subjective impression of reward deficit triggered by unfavourable external circumstances. This may be connected with a critical assessment of one's own behaviours, with a fear of making bad decisions, of not being in accordance with a self-ideal, or of having to face potential negative outcomes (Shalev \& Sulkowski, 2009). Despite its theoretical and clinical interest, the relationship between SR, adverse experiences and OCS has to be interpreted with caution because a high level of SR may intrinsically include a propensity to act out (captured, for instance, by SR items like 'Do you like displaying your physical abilities even though this may involve danger?'). Thus, it cannot be excluded that individuals reporting a high score of SR may, at the same time, be more inclined to put themselves in potentially dangerous circumstances. The design of the current study prevents any interpretation of the causal role of SR, trauma and OCS. Aside from a longitudinal study design, research distinguishing between 'ideal' and 'acting out' aspects of SR may shed light on this issue in the future.

Finally, we have to acknowledge that the current PDS-F checklist is limited to events that generally occur during adulthood. Lochner et al. (2007) observed that in a group of 83 OCD patients, OCS severity was associated with self-reported accounts of child neglect and abuse. Interestingly, Mathews et al. (2008) demonstrated that childhood trauma, particularly emotional neglect, plays a role in the expression of dysfunctional levels of OCS in students, possibly through a personality

\section{Behaviour Change}

Downloaded from https:/www.cambridge.org/core. University of Basel Library, on 30 May 2017 at 16:29:06, subject to the Cambridge Core terms of use, available at https:/www.cambridge.org/core/terms. https://doi.org/10.1375/bech.28.2.75 
trait of consciousness. This finding raises two important concerns. On the one hand, it suggests that further studies on OCS should integrate an extensive assessment of childhood adverse experiences. On the other hand, it indicates that other personality dispositions than the ones we studied (SP, SR) should be informative in delineating the boundaries of a diathesis-stress model of OCS.

This study presents limitations that preclude its generalisation. First, the absence of a longitudinal design rules out the possibility to draw firm conclusions about causality. Second, our sample was mainly constituted by females. More representative gender recruitment would allow conclusions about possible gender effects. Third, our results are drawn on a nonclinical sample. Even if this is current practice (Mataix-Cols, et al., 2000; Muris et al., 1997), direct generalisations to clinical OCD patients are not possible before formal replications with OCD patients. Four, the present study relies on self-report measures that can be biased by social-desirability and awareness accessibility. These limits can be critical in the assessment of traumatic and affective phenomena that deserve more indirect measures (like, for instance, behaviour observation). Finally, our measure of adverse event exposure includes items of physical and sexual abuse without specifically identifying childhood maltreatment that has previously been shown to be of interest in OCD (Lochner et al., 2002, 2007; Mathews et al., 2008). Despite these limitations, the current study sheds new light on a diathesis-stress model of OCS which addresses the complex role of dispositional-situational interactions. Future work is obviously needed to precisely determine the interplay of these factors in the onset and maintenance of OCS subgroups over time.

\section{References}

Bartz, J.A., \& Hollander, E. (2006). Is obsessive-compulsive disorder an anxiety disorder? Progress in Neuro-Psychopharmacology $\mathbb{E}$ Biological Psychiatry, 30, 338-352.

Beck, A.T., Steer, R.A., \& Brown, G.K. (1998). Inventaire de Dépression de Beck - 2ème édition (BDI-II) (Version 2). Paris: Editions du Centre de Psychologie Appliquée (ECPA).

Bleuler, J., \& Freud, S. (1893-95). Studies on hysteria. In J. Strachey (Ed.), The Standard Edition (Vol. 2, pp. 215-222). London: Hogarth Press.

Coles, M.E., \& Horng, B. (2006). A prospective test of cognitive vulnerability to obsessive-compulsive disorder. Cognitive Therapy and Research, 30, 723-734.

Coles, M.E., Pietrefesa, A.S., Schofield, C.A., \& Cook, L.M. (2008). Predicting changes in obsessive compulsive symptoms over a six-month follow-up: A prospective test of cognitive models of obsessive compulsive disorder. Cognitive Therapy and Research, 32, 657-675.

Cromer, K.R., Schmidt, N.B., \& Murphy, D.L. (2007a). Do traumatic events influence the clinical expression of compulsive hoarding? Behaviour Research and Therapy, 45, 2581-2592.

Cromer, K.R., Schmidt, N.B., \& Murphy, D.L. (2007b). An investigation of traumatic life events and obsessive-compulsive disorder. Behaviour Research and Therapy, 45, 1683-1691.

David, M., Ceschi, G., Billieux, J., \& Van der Linden, M. (2008). Depressive symptoms after 84 trauma: Is self-esteem a mediating factor? The Journal of Nervous and Mental Disease, 196, $735-742$.

Dawe, S., Gullo, M.J., \& Loxton, N.J. (2004). Reward drive and rash impulsiveness as dimensions of impulsivity: Implications for substance misuse. Addictive Behaviors, 29, 1389-1405.

De Silva, P., \& Marks, M. (2001). Traumatic experiences, post-traumatic stress disorder and obsessive-compulsive disorder. International Review of Psychiatry, 13, 172-180.

Dohrenwend, B.S., \& Dohrenwend, B.P. (1981). Stressful life events and their contexts. New York: Neale Watson Academic Publications. 
Dugas, M.J., Buhr, K., \& Ladouceur, R. (2004). The role of intolerance of uncertainty in etiology and maintenance of GAD. In R. G. Heimberg, C.L. Turk \& D.S. Mennin (Eds.), Generalized anxiety disorder: Advances in research and practice (pp. 143-163). New York: Guilford.

Dugas, M. J., \& Robichaud, M. (2007). Cognitive-behavioural treatment for generalized anxiety disorder: From science to practice. New York: Routledge.

Elliot, A.J., \& Thrash, T.M. (2002). Approach-avoidance motivation in personality: Approach and avoidance temperaments and goals. Journal of Personality and Social Psychology, 82, 804-818.

Enticott, P.G., \& Ogloff, J.R.P. (2006). Elucidation of impulsivity. Australian Psychologist, 41, 3-14.

Foa, E.B., Cashman, L., Jaycox, L., \& Perry, K. (1997). The validation of a self-report measure of posttraumatic stress disorder: The Posttraumatic Diagnostic Scale. Psychological Assessment, 9, $445-451$.

Foa, E.B., Huppert, J.D., Leiberg, S., Langner, R., Kichic, R., \& Hajcak, G. (2002). The ObsessiveCompulsive Inventory: Development and validation of a short version. Psychological Assessment, 14, 485-596.

Freeston, M.H., Rhéaume, J., Letarte, H., Dugas, M.J., \& Ladouceur, R. (1994). Why do people worry? Personality and Individual Differences, 17, 791-802.

Frost, R.O., \& Steketee, G. (1997). Perfectionism in obsessive-compulsive disorder patients. Behaviour Research and Therapy, 35, 291-296.

Fullana, M.A., Mataix-Cols, D., Caseras, X., Aloso, P., Menchon, J.M., Vallejo, J., \& Torrubia, R. (2004). High sensitivity to punishment and low impulsivity in obsessive-compulsive patients with hoarding symptoms. Psychiatry Research, 129, 21-27.

Fullana, M.A., Mataix-Cols, D., Trujillo, J.L., Caseras, X., Serrano, F., Aloso, P.,...Torrubia, R. (2004). Personality characteristics in obsessive-compulsive disorder and individuals with subclinical obsessive-compulsive problems. British Journal of Clinical Psychology, 43, 387-398.

Gay, P., Rochat, L., Billieux, J., d'Acremont, M., \& Van der Linden, M. (2008). Heterogeneous inhibition processes involved in different facets of self-reported impulsivity: Evidence from a community sample. Acta Psychologica, 129, 332-339.

Grabe, H.J., Ruhrmann, S., Spitzer, C., Josepeit, J., Ettelt, S., Buhtz, F.,...Freyberger, H.J. (2008). Obsessive-compulsive disorder and posttraumatic stress disorder. Psychopathology, 41, 129-134.

Gray, J.A., \& McNaughton, N. (2000). The neuropsychology of anxiety: An enquiry into the functions of the septo-hippocampal system. Oxford, UK: Oxford University Press.

Hearn, M., Ceschi, G., Brillon, P., \& Van der Linden, M. (2010). A French adaptation of the Posttraumatic Diagnostic Scale (PDS-F). Submitted.

Huppert, J.D., Moser, J.S., Gershuny, B.S., Riggs, D.S., Spokas, M., Filip, J.,...Foa, E.B. (2005). The relationship between obsessive-compulsive and posttraumatic stress symptoms in clinical and non-clinical samples. Journal of Anxiety Disorders, 19, 127-136.

Lardi, C., Billieux, J., d'Acremont, M., \& Van der Linden, M. (2008). A French adaptation of a short version of the Sensitivity to Punishment and Sensitivity to Reward Questionnaire (SPSRQ). Personality and Individual Differences, 45, 722-725.

Lochner, C., du Toit, P., Zungu-Dirwayi, N., Marais, A., Van Kradenburg, J., Curr, B.,...Stein, D.J. (2002). Childhood trauma in obsessive-compulsive disorder, trichotillomania, and controls. Depression and Anxiety, 15, 66-68.

Lochner, C., Seedat, S., Hemmings, S.M.J., Moolman-Smook, J.C., Kidd, M., \& Stein, D.J. (2007) Investigating the possible effects of trauma experiences and 5-HTT on the dissociative experiences of patients with OCD using path analysis and multiple regression. Neuropsychobiology, 56, $6-13$.

Mataix-Cols, D., Rosario-Campos, M.C., \& Leckman, J.F. (2005). A multidimensional model of obsessive-compulsive disorder. American Journal Psychiatry, 162, 228-238.

Mataix-Cols, D., Vallejo, J., \& Sanchez-Turet, M. (2000). The cut-off point in sub-clinical obsessive-compulsive research. Behavioural and Cognitive Psychotherapy, 28, 225-233.

Mathews, C.A., Kaur, N., \& Stein, M.B. (2008). Childhood trauma and obsessive-compulsive symptoms. Depression and Anxiety, 25, 7742-7751.

\section{Behaviour Change}

Downloaded from https:/www.cambridge.org/core. University of Basel Library, on 30 May 2017 at 16:29:06, subject to the Cambridge Core terms of use, available at https:/www.cambridge.org/core/terms. https://doi.org/10.1375/bech.28.2.75 
Muris, P., Merckelbach, H., \& Clavan, M. (1997). Abnormal and normal compulsions. Behaviour Research and Therapy, 35, 249-252.

Myers, S.G., Fisher, P.L., \& Wells, A. (2009). Metacognition and cognition as predictors of obsessive-compulsive symptoms: A prospective study. International Journal of Cognitive Therapy, 2, 132-142.

Nestadt, G., Samuels, J., Riddle, M.A., Liang, K.-Y., Bienvenu, O.J., Hoehn-Saric, R....Cullen, B. (2001). The relationship between obsessive-compulsive disorder and anxiety and affective disorder: Results from the John Hopkins OCD family study. Psychological Medicine, 31, 481-487.

Pietrefesa, A.S., \& Coles, M.E. (2008). Moving beyond an exclusive focus on harm avoidance in obsessive compulsive disorder: Considering the role of incompleteness. Behaviour Therapy, 39, 224-231.

Rachman, S. (1993). Obsessions, responsibility, and guilt. Behaviour Research and Therapy, 31, 149-154.

Rachman, S. (1997). A cognitive theory of obsessions. Behaviour Research and Therapy, 35, 793-802.

Ray Li, C.-S., \& Chen, S.-H. (2007). Obsessive-compulsiveness and impulsivity in a non-clinical population of adolescent males and females. Psychiatry Research, 149, 129-138.

Salkovskis, P. (1999). Understanding and treating obsessive-compulsive disorder. Behaviour Research and Therapy, 37, S29-S52.

Salkovskis, P., Shafran, R., Rachman, S., \& Freeston, M.H. (1999). Multiple pathways to inflated responsibility beliefs in obsessional problems: Possible origins and implications for therapy and research. Behavioural Research and Therapy, 37, 1055-1072.

Samuels, J., Nestadt, G., Bienvenu, O.J., Costa, P.T., Riddle, M.A., Liang, K.-Y.,...Cullen, B.A.(2000). Personality disorders and normal personality dimensions in obsessive-compulsive disorder. British Journal of Psychiatry, 177, 457-462.

Sasson, Y., Dekel, S., Nacasch, N., Chopra, M., Zinger, Y., Amital, D., \& Zohar, J. (2005). Posttraumatic obsessive-compulsive disorder: A case series. Psychiatry Research, 135, 145-152.

Shalev, I., \& Sulkowski, M.L. (2009). Relations between distinct aspects of self-regulation to symptoms of impulsivity and compulsivity. Personality and Individual Differences, 47, 84-88.

Storch, E., Abramowitz, J.S., \& Goodman, W. (2008). Where does obsessive-compulsive disorder belong in DSM-V? Depression and Anxiety, 25, 336-347.

Sulkowski, M.L., Jordan, C., Reid, A., Graziano, P.A., Shalev, I., \& Storch, E. (2009). Relations between impulsivity, anxiety, and obsessive-compulsive symptoms in a non-clinical sample. Personality and Individual Differences, 47, 620-625.

Summerfeldt, L.J., Hood, K., Antony, M.M., Richter, M.A., \& Swinson, R.P. (2004). Impulsivity in obsessive-compulsive disorder: Comparisons with other anxiety disorders and within ticrelated subgroups. Personality and Individual Differences, 36, 539-553.

Torrubia, R., Avila, C., Molto, J., \& Caseras, X. (2001). The Sensitivity to Punishment and Sensitivity to Reward Questionnaire (SPSRQ) as a measure of Gray's anxiety and impulsivity dimensions. Personality and Individual Differences, 31, 837-862.

Tuerk, P.W., Grubaugh, A.L., Hamner, M.B., \& Foa, E.B. (2009). Diagnosis and treatment of PTSDrelated compulsive checking behaviors in veterans of Iraq war: The influence of military context on the expression of PTSD symptoms. American Journal of Psychiatry, 166, 762-767.

Van der Linden, M., Ceschi, G., Zermatten, A., Dunker, D., \& Perroud, A. (2005). Investigation of response inhibition in obsessive-compulsive disorder using the Hayling task. Journal of the International Neuropsychological Society, 11, 776-783.

Zermatten, A., \& Van der Linden, M. (2008). Impulsivity in non-clinical persons with obsessivecompulsive symptoms. Personality and Individual Differences, 44, 1824-1830.

Zermatten, A., Van der Linden, M., Jermann, F., \& Ceschi, G. (2006). Validation of a French version of the Obsessive-Compulsive Inventory-Revised in a non-clinical sample. European Review of Applied Psychology, 56, 151-155. 